April 29, $2015 \quad 8: 28$ WSPC/INSTRUCTION $\quad$ FILE

'2013.IJHR' DiazetalV3. CameraReady 02"

International Journal of Humanoid Robotics

(C) World Scientific Publishing Company

\title{
EVALUATING GROUP-ROBOT INTERACTION IN CROWDED PUBLIC SPACES: A WEEK-LONG EXPLORATORY STUDY IN THE WILD WITH A HUMANOID ROBOT GUIDING VISITORS THROUGH A SCIENCE MUSEUM
}

\author{
MARTA DÍAZ, DENNYS PAILLACHO*, CECILIO ANGULO \\ Technical University of Catalonia, Victor Balaguer 1 \\ 08800 Vilanova i la Geltrú, Spain \\ \{marta.diaz, dennys.paillacho, cecilio.angulo\}@upc.edu \\ * Escuela Superior Politécnica del Litoral, Km 30.5 vía Perimetral \\ 09015863 Guayaquil, Ecuador \\ dpaillac@espol.edu.ec \\ ORIOL TORRES, JONATHAN GONZÁLEZ \\ PAL Robotics, Pujades 77-79 \\ 08005 Barcelona, Spain \\ \{oriol.torres, jon.gonzalez\}@pal-robotics.com \\ JORDI ALBO-CANALS \\ La Salle BCN - Ramon Llull University, Quatre Camins 30 \\ 08022 Barcelona, Spain \\ jalbo@salle.url.edu
}

\begin{abstract}
This paper describes an exploratory study on group interaction with a robot-guide in an open large-scale busy environment. For an entire week a humanoid robot was deployed in the popular Cosmocaixa Science Museum in Barcelona and guided hundreds of people through the museum facilities. The main goal of this experience is to study in the wild the episodes of the robot guiding visitors to a requested destination focusing on the group behavior during displacement. The walking behavior follow-me and the face to face communication in a populated environment are analyzed in terms of guidevisitors interaction, grouping patterns and spatial formations. Results from observational data show that the space configurations spontaneously formed by the robot guide and visitors walking together did not always meet the robot communicative and navigational requirements for successful guidance. Therefore additional verbal and nonverbal prompts must be considered to regulate effectively the walking together and follow-me behaviors. Finally, we discuss lessons learned and recommendations for robot's spatial behavior in dense crowded scenarios.
\end{abstract}

Keywords: Group-robot interaction; robotic-guide; social navigation; space management; spatial formations; group walking behavior; crowd behavior. 


\section{Introduction}

Service robots are increasingly taking part of people daily life activities interacting socially and sharing spaces with individual and groups in close proximity. For social robots featured with walk around functionality, key questions to be addressed for effective performance are how to move (i.e. speed, kind of movement, trajectories), where to perform (i.e. proximity management) and how to place (i.e. distance, position, stance and orientation) to be unobtrusive, effective and socially congruent.

Promising attempts to optimize social robots spatial management in different scenarios (e.g. assistive telepresence at home) have been developed applying models and knowledge from social psychology (i.e. proxemics, space formations, group walking patterns and crowd dynamics). Guidance is one of the most useful services of robots in public spaces as museums, exhibitions, malls, and tourist sites. Assuming the role of guide the robot not only provides people with appropriate information to make the visit a more enjoyable experience but help them to reach intended destinations. The main difference between an informer or recommender robot and a robotic guide is that guidance in public spaces implies social navigation in a highly dynamic scenario; i.e., that the social robot navigation design must consider the actions of people around them.

Socially compliant navigation ${ }^{1}$ implies planning and performing robot's trajectories and motion behavior taking into account the communicative function and social rules of space management in a shared location. Smart spatial behavior (e.g. interpersonal distance, orientation) according to social norms would not only enhance collocated user's safety and acceptance but also provide mobile robots with an intuitive rich nonverbal channel to communicate intentions (e.g. shift direction, initiate displacement) and to express emotional content ${ }^{2}$.

To explore guide robot-visitors performance in open large-scale dense environments PAL Robotics' REEM robot was deployed during a week in the CosmoCaixa Science Museum informing, motivating, giving directions and walking groups of visitors to requested locations. The whole experience was video-recorded by two external general-view cameras and one on-board camera for observational data analyses. Our approach is to put the focus on the group spatial behavior rather than on individuals taken as independent agents. Therefore, in this paper visitors' group behavior while walking (i.e. spatial arrangement) will be described and analyzed -based on the knowledge on group walking and crowd dynamics-, as well as communicative behavior towards the robot. Lessons learned from this long lasting experiment in the wild could also be considered for designing spatial behavior of mobile service robots in other contexts as receptions, leisure parks or hospitals.

In the next section findings from previous work on guide-robots in open largescale environments and related knowledge from the fields of proxemics and group walk are reviewed. In Section 3, the experience at CosmoCaixa Science Museum Barcelona is described. Next, analysis of recorded human-robot social interaction data is detailed with special focus on the follow-me episodes. A discussion and 
recommendations for improved experience design follow in Section 6. Finally, some conclusions are provided and further research is pointed out.

\section{Related Work}

Mobile service robots may deploy their activity in close proximity to people either in closed (e.g. home ${ }^{3}$, school ${ }^{4}$, office, nursing home ${ }^{5}$ ) and open environments (e.g. exhibitions $^{6}$, museums ${ }^{7,8,9}$, malls). In closed environments the occupants are known, and often belong to few homogeneous profiles (e.g. ages, familiarity to technology). On the other hand, in open public spaces occupants are unknown, diverse, variable and dynamic, often including heterogeneous profiles (i.e. teenagers, staff, elderly). A frequent situation in large-scale open public environments is the configuration of dense crowds that the robot is supposed to travel through fulfilling safety (the primary requirement of a robot operating in a public space), reliability and social requirements at a time.

Moreover, robots with the "walk around" functionality get involved in spatial relationships with people ${ }^{3,10}$. Spatial relationships are a combination of distance, relative position and orientation that occur naturally whenever two or more people engage in an interaction ${ }^{11}$ and convey significant and relevant social information (e.g. how each of them is involved) and also define an interpersonal space for developing activity.

Empirical studies in telepresence applications have identified the management of spatial relationships between people and robot as a main issue in order to improve the quality of interaction taking into account that interpersonal distances convey significant and relevant social information ${ }^{10}$. Based on Kendon's model ${ }^{12}$, the authors identify space formations or spatial patterns (e.g. vis-a-vis, side-by-side, L-shape, follow or ahead) related to the roles adopted by the robot, the activities and the spatial constrains, as well as individual variables such as familiarity with the agent. As a conclusion, when physical constraints (e.g., narrow passages) along with navigational requirements prevents the robot to maintain the convenient spatial behavior, it can compensate this situation with other interactive behaviors (e.g. verbally apologizing for an inappropriate distance or reducing the eye-contact) to maintain an overall degree of desired intimacy.

An open public scenario where autonomous mobile robots have been deployed are museums. Three aspects make the robot navigation in a museum specially difficult: the robot has to guide visitors through dense even crowded spaces, some elements of the physical space could be "invisible" to the robot (e.g. glass walls) and the configuration of the environment change frequently (e.g. pieces of furniture, fences). The robot guide in a museum faces two primary challenges: navigating safely, reliably and socially through crowds, and interact with people in a compelling and intuitive way ${ }^{7}$.

Guidance is a demanding collaborative task that requires communicating intentions $^{13}$ (i.e. robot offers the service, visitors select a destination and request 
the "Bring me there" function, the robot heads towards the destination) and social navigation (i.e. walk together to the target location). Walking along following the leader implies complex space regulations (i.e. distancing, spatial configurations) to allow guide and visitors group up and walk together effectively. These space relationships during guidance must be at a time socially meaningful and compatible with the robot's navigation specifications (i.e. collision avoidance performance ${ }^{14}$ ).

To model the navigation through crowds of dynamic agents with uncertain trajectories some attempts has been done drawing inspiration from the pedestrians behaviors in dense environments, where people usually engage in "joint collision avoidance" (called the social forces model) and adapt their trajectories to each other to make room for navigation ${ }^{15}$. This model is proposed to overcome shortcomings of models based on anticipate trajectories taking each individual as independent agents that often lead when tested in the wild to ineffective overcautions robot behaviors and even to "freezing the robot" when people attracted by the robot surround it and once the environment surpasses a certain level of complexity, the planner decides that all forward paths are unsafe and freezes in place to avoid collisions. In the case of the "freezing problem", the focus on group collaborative behavior rationales can be more fruitful to design robot's ability to elicit the natural cooperative behavior of making room to create feasibly trajectories. Verbal and nonverbal cues as look at the intended direction or asking for permission could be enough to make room for safe navigation.

Communication between robot and users in this scenario is complex. According to its role, naturally the guide communicates with dynamic groups of different sizes, densities and composition often walking around in busy environments. Thus, the simpler models of one-to-one and face to face human-robot interaction are largely surpassed in this context. Moreover, in the social situation of visiting a museum (as an entertainment venue) people are likely to be curious, active and attracted by new appealing things as the robot itself. Exploration of the robot and of its limits ${ }^{16}$ is a natural behavior that sometimes lead to malfunction (e.g. push the emergeny stop button) and even to damage the robot seriously. In addition to unintentional damage, malicious behaviors towards service robots are also been reported, making robots' robustness or even resilience a key specification in public spaces. ${ }^{8}$. Several robotic museum guides as Minerva ${ }^{17}$, Robovie ${ }^{5}$, RoboX ${ }^{6}$, Rhino $^{7}$, Chips, Sweetlips, Joe And Adam40-80 ${ }^{8}$ do quite well in addressing people and keeping their attention, however interaction between robots and humans is still limited due to the highly challenging environment. As far as we know, research on robotic guides has mainly focused on verbal and non-verbal communicative behaviors (i.e. dialog) to improve the visitor experience in static situations rather than on the spatial arrangements during guidance.

On the other hand, although there are several studies that evaluate the HRI by spatial relationships, these are framed only in the individual and in closed and non-natural environments ${ }^{3,10,18,19,20,21}$. An interesting approach related to spatial 
relationships, buy in crowds of pedestrians, was conducted by Bandini et.al. ${ }^{22}$. In this work, Bandini analyzes the behavior of groups such as the characteristics of the groups and their group spatial arrangement while walking in dynamic environments. From an empirical research, different patterns of group spatial arrangement (e.g. line- abreast, v-pattern and river-like) and its significance in relation to the social cohesion of the group were analyzed.

The present study focus on the description of follow-me group behavior from observational data gathered in a naturalistic trial in the wild, applying models from group spatial management (i.e. proxemics, group walking ${ }^{23}$ and crowd dynamics ${ }^{22}$.

\section{The Experience at Cosmocaixa Museum}

This section discusses general issues related to the design and development of the experience at CosmoCaixa Science Museum Barcelona: our main objective, the employed service robot, the scenario, and the task.

\subsection{Design}

During 6 days the REEM robot was deployed in a restricted area in the CosmoCaixa Museum navigating autonomously around the facility. The robot played the role of a museum guide offering information and guidance to visitors when requested.

The presence of researchers and technical staff was reduced to a discrete and permanent remote surveillance of the robot's performance. The intervention of technical staff was aimed at recovering the robot for eventual breakdowns and discouraging misuse to enhance people safety and to prevent robot's damage.

No briefing or instruction was given to visitors and no adaptation of the physical environment was implemented except from the two cameras placed in the walls in an effort to maximize the study ecological validity preserving the natural every-day conditions and routines in the Museum activity (i.e. density and flows of visitors).

\subsection{Objective}

The main goal is to investigate in the wild visitors-robot guide interaction while walking (i.e. space formations) and in face-to-face communication (i.e. natural and computer-based interactive behavior).

A first set of research questions are related to robot effectiveness according to its role: Under which conditions and to what extend does the robot attract and entertain visitors, engage people in satisfactory face-to-face interaction, help people to find their way? A second set of research questions are about group spatial management during guidance: Can patterns of spatial group behavior during group walking be identified? Are they similar to those modeled for human groups? Are all of them equally suitable for effective guiding? Do visitors' individual variables influence the group walking performance? Do group size and/or composition influence the space arrangements? 
The ultimate purpose is to guide the redesign of robot's interactive behavior and -as far as possible in an exploratory study- to draw new knowledge about spatiality and interactive behavior in group-robot interaction.

\subsection{The robot}

REEM is a $1,65 \mathrm{~m}$ high humanoid robot with 22 degrees of freedom. The upper part of the robot comprises of a torso with a touchscreen, two motorized arms, which give it a high degree of expression, and a head, which is also motorized (Figure 1). The robot features a rear small platform which can be used to transport objects (e.g. a trolley). The mobile base contains a lithium battery that provides up to eight hours of autonomous operation. A complete range of sensors (i.e. cameras, ultrasonic, lasers) support dynamic distancing and collision avoidance for a safe navigation $^{\mathrm{a}}$.

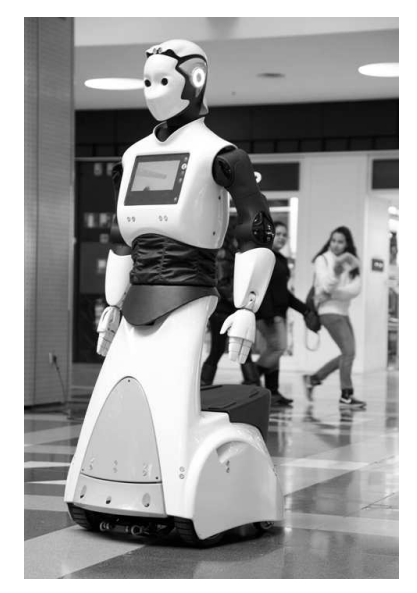

Fig. 1: REEM robot from PAL Robotics.

As a sophisticated anthropomorphic robot, REEM features diverse elements and devices to support verbal and non-verbal communication. Some of them are recognizable mechanic versions of natural-like elements as eyes -that are just two holes in the face without lids, eyelids or pupils-framed under the shape of brows. The monochromatic white face presents as well the shape of a nose but no mouth is represented. At both sides of the head are placed two elements evoking vaguely the position and shape of ears that are enlightened when the robot is activated. The head can move up and down and turn right and left and so does the torso. The articulated arms and hands may support social and utilitarian behavior (i.e. shake 
hands, point, wave, grasp). In addition users can interact with REEM through the friendly-use 12 inch touchscreen interface on the robot's torso where ad hoc interactive multimedia applications can be run.

Therefore, according to REEM appearance and features enriched intuitive human-like non-verbal communication can be implemented through head and body movement (i.e. gaze behavior), posture (i.e. orientation) and smart navigation (i.e. social distancing) (see Table 1).

Table 1: REEM's potential interactive behavior.

\begin{tabular}{llll}
\hline Dimensions & Variables & Categories & Subcategories \\
\hline Verbal & Spoken & Unidirectional & non conversational \\
\hline Non Verbal & Gaze behavior & Eye contact & \\
& & Look at & \\
& Gestures & Head movements & \\
& & Arms motion & \\
& Hands motion & \\
& Body stance & & Direction \\
& Displacement & Social navigation & Velocity \\
& & & Follow \\
& & & Guide \\
& & & Distacle Avoidance \\
\hline
\end{tabular}

For safety and feasibility issues the use of some REEM's interactive resources was deliberately constrained during the autonomous operation. Consequently the robot's potentiality for verbal and non-verbal communication was reduced to notfacial/not-verbal behavior ${ }^{2,24}$. Specifically, arms and hands were blocked and stuck to the body for safety issues. Not conversational communication is implemented but the information displayed on the screen was as well spoken out by the robot as redundant feedback during face-to-face interaction. Therefore, in our study we investigate to which extend REEM is capable to carry out the main role-dependent functions of smart guiding based only on head movements, motion and interfacebased interaction.

\subsection{Scenario and setup}

CosmoCaixa is a science museum located in Barcelona, Spain. The museum hosts a very popular planetarium and a wide range of permanent and temporary exhibitions and attractions where visitors, mainly children and their families, are encouraged 
to experience and interact actively with the environment. Up to 800,000 people visited the Museum in $2012^{25}$. In 2006 CosmoCaixa Barcelona was awarded by the 'European Museum of the Year Award' -institution sponsored by the European Council- as the best science museum in Europe.

The field study was carried out from Tuesday November 27th to Sunday December 2nd, 2012 on the occasion of the European Robotics Week. This 6 days schedule includes a free entrance day (Sunday) when the number of visitors increases considerably.

The robot was deployed in the floor -2 in a restricted area of about 5 meters wide and 40 meters long in a centric corridor leading to the more popular facilities (see Figure 2 and Figure 3). Three locations ( $A, B$ and $C$ in Figure 2) were defined as the three possible destinations. Visitors going to "Planetarium" and "Flooded Forest" were walked to $A$, visitors going to "Flash" and "Touch-Touch!" to $B$ and visitors to the activity "Clik" to $C$. Point $D$ is the initial location of the robot close to one of the main entrances and besides an information desk.

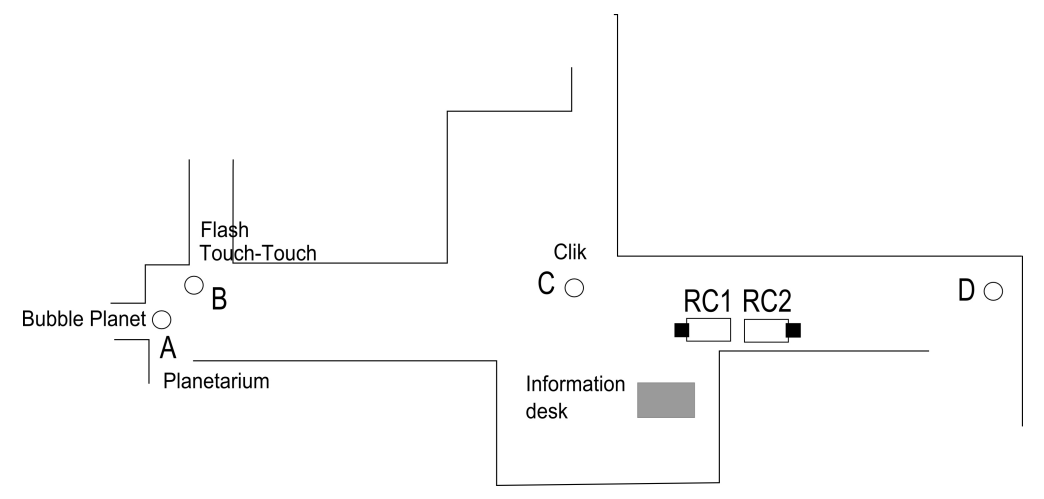

Fig. 2: Map for the robot placement at CosmoCaixa Science Museum Barcelona

\subsection{Task}

According to its role the general function of REEM is to enrich visitors' experience by exhibiting itself as an attraction, providing entertainment and information and eventually bringing visitors to requested destinations. The robot role is deployed in three activities: offering services, face-to-face interaction and guidance (Figure 4).

The purpose of offering services is to attract people to engage in interaction. This phase starts as soon as the robot is activated, the ears' lights turn on and the home page is displayed on the touchscreen at the robot's chest. According to the programmed mode, the robot either deploy a proactive behavior moving around among visitors or remain stationary by the information desk until someone eventually approach. This phase ends when the screen is touched and the interactive 


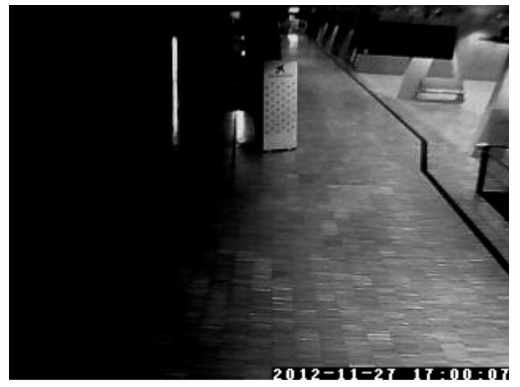

(a)

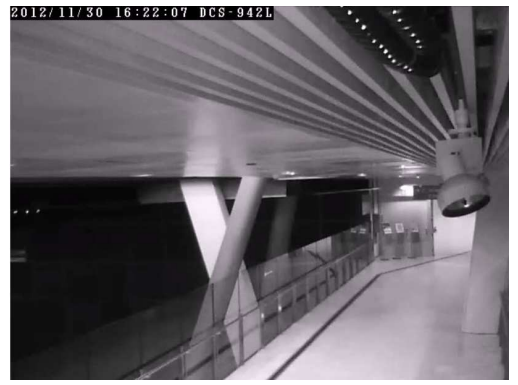

(b)

Fig. 3: External camera shots: (a) from recording camera 1 (RC1); and, (b) from recording camera $2(\mathrm{RC} 2)$.

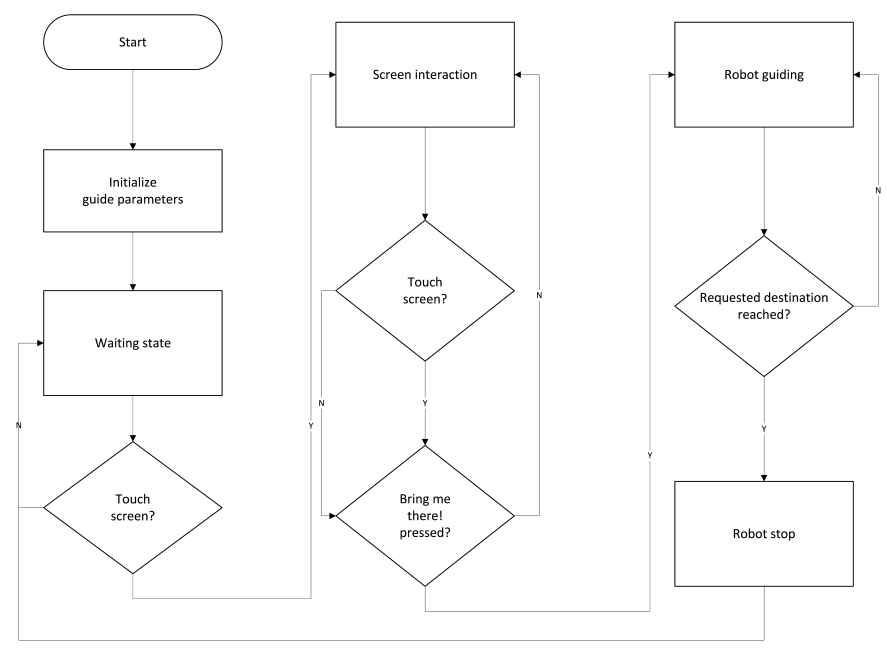

Fig. 4: Flow of robot's guide role.

multimedia application is launched. During face-to-face interaction the communication is mediated by the graphic interface. The textual information displayed on the screen is spoken aloud redundantly by the robot to enhance robot's social presence. A tree of the easy-to-use application architecture is shown in Figure 5. If the visitor selects the option Bring me there! the robot initiates the guidance navigating to the target location associated to the requested destination. Once the target location is reached or the mission definitive aborted (e.g. the robot is blocked by a crowd) the robot stops and restarts the activity from the first phase (i.e. offering services). 
April 29, $2015 \quad 8: 28 \quad$ WSPC/INSTRUCTION $\quad$ FILE

'2013.IJHR' DiazetalV3. CameraReady 02"

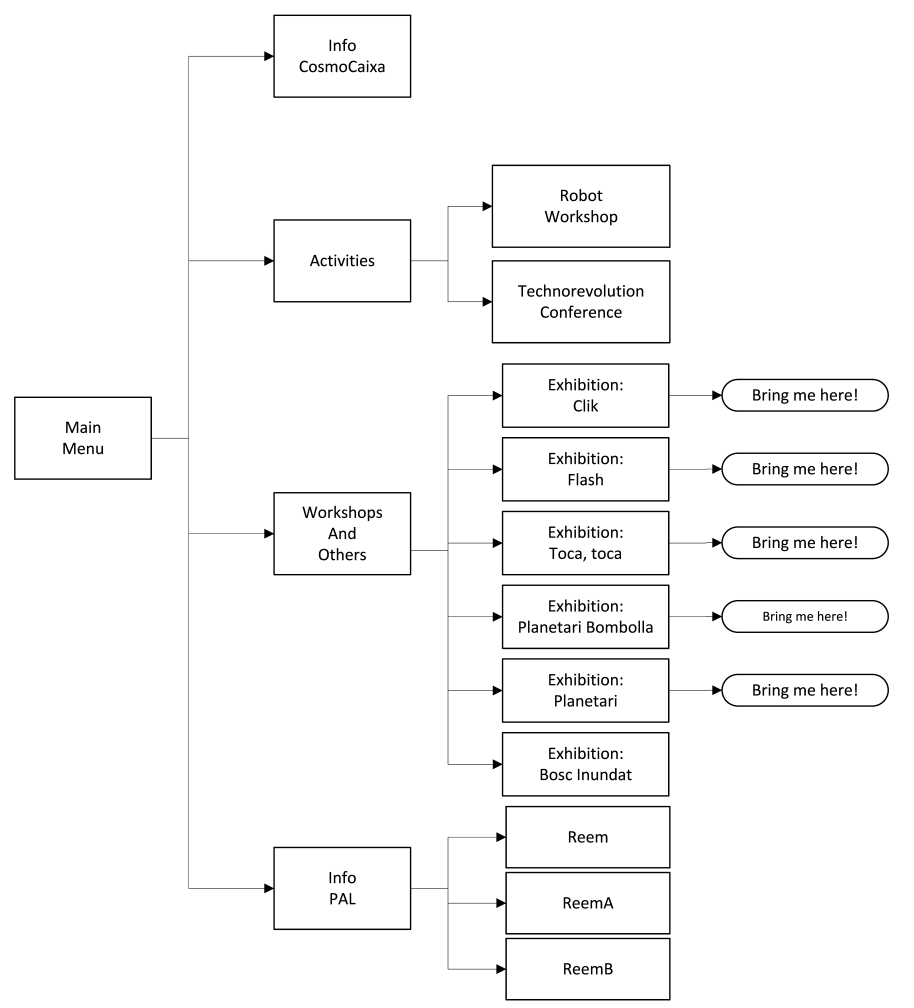

Fig. 5: Architecture of the multimedia GUI interface.

\subsubsection{Data collection}

To register continuously visitors and robot activity two commercial surveillance cameras (RC1 and RC2 in Figure 2) were set in the center of the corridor fixed to the building pillars at a height of approximately $3 m$ to have an aerial overview of the experimental area (Figure 3). In addition, the robot's on-board camera (RC3) placed behind the robot's eyes was used to obtain a close-up view of visitors from the robot's perspective to study face-to-face interaction. The three video sources were downloaded and stored daily for further processing and analysis.

\section{Analyses}

\subsection{Observational data processing}

According to the study's aim the spatial arrangements performed during guidance and visitors' face-to-face communicative behavior with the robot were analyzed. In Table 2 the dimensions for group characterization and the coding scheme for group walking behavior and interactive face-to-face behavior are summarized. 
April 29, $2015 \quad 8: 28$ WSPC/INSTRUCTION $\quad$ FILE

''2013'IJHR' DiazetalV3. CameraReady 02"

The coding was carried out manually by two of the experimenters working together.

Table 2: Group characterization and Visitor's behaviors.

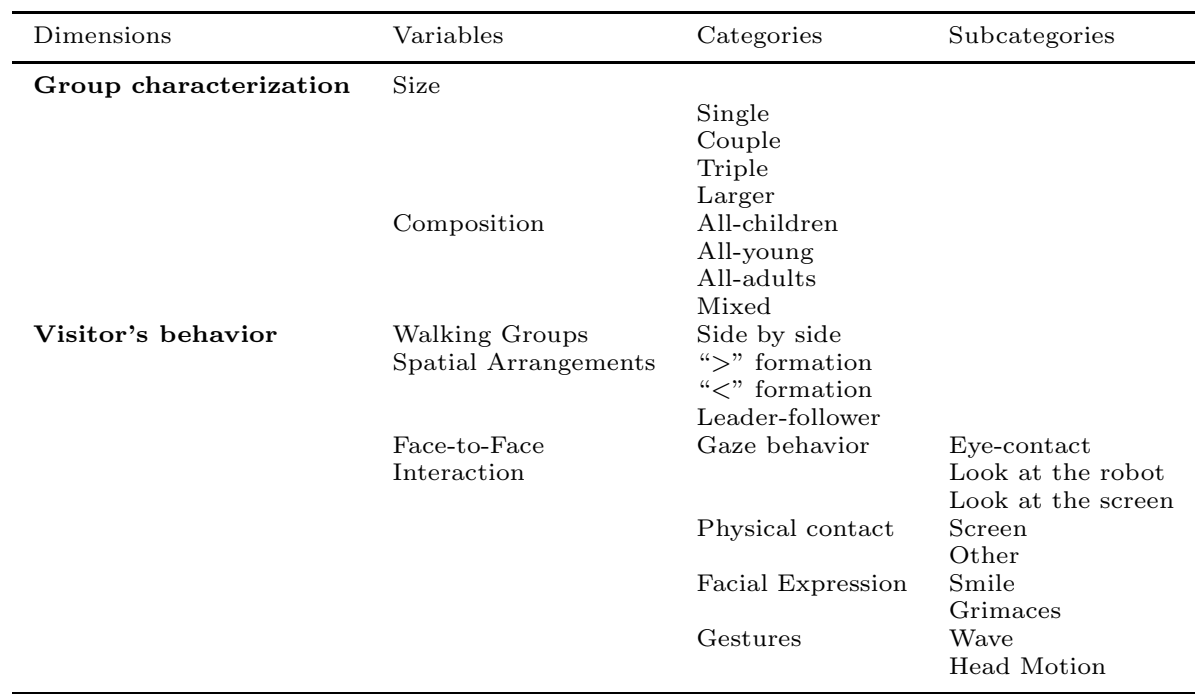

\subsubsection{Follow-me episodes}

To improve the row data from the panoramic cameras and to select the relevant episodes of guidance a preliminary preprocessing of the videos was done as follows:

- Up to 4828 minutes of recordings from external cameras (RC1 and RC2 in Figure 2) were labeled and stored.

- Recordings without any kind of movement were eliminated using computer vision techniques resulting in a total duration of 3966 minutes.

- The sequences where the robot appeared simultaneously with at least one person (i.e. visitors or museum staff) were selected. As a result, a total of 283 scenes with approximately 825 minutes of total duration were selected.

- Every episode of guidance were selected resulting in 91 clips with approximately 96 minutes of total time.

\subsubsection{Face-to-face sequences}

83 minutes of video were registered by the on-board camera (RC3 in Figure 2) to analyse people's face-toface behavior interacting with the robot. 14 episodes were pre-selected for a total duration of $47 \mathrm{~min}$. In order to obtain quantitative data, 
the 14 episodes were sampled considering 10 seconds every minute obtaining a set of 47 sequences with a total time of 8 minutes approximately.

\subsubsection{Groups}

Visitors' groups in the 91 episodes of guidance are characterized by composition (i.e. age of the members) and size. A walking group in guidance is composed by the robot and the visitors that move along with it regardless the relative distance and position between them. Visitors that join the group on the fly -and that probably are not aware where are the robot heading to- are also considered members of the group.

In face-to-face interaction, social context is defined by all the people that are in camera and within the social space at any moment during the sequence regardless the distance, position, orientation or behavior. Co-present individuals beyond the social distance are only considered when they look at the robot at least once during the sequence.

\subsubsection{Group spatiality during guidance}

In this study a guidance episode is the sequence of walking together behavior deployed by the robot and a group of visitors starting when the "Bring me there" option is selected on the screen and ending when the robot stops and comes back to offering services state. Guidance ends either when the robot reach the requested destination or when the robot's trajectory is definitely aborted (e.g. robot blocked by a crowd of visitors, robot stuck in a corner, emergency shutdown). Temporary stops during the displacement due to navigational constraints (i.e. mobile obstacles avoidance) do not end the guidance sequence provided the trajectory is resumed by the robot. Any displacement of visitors along with the robot is considered a guidance episode regardless to the particular relative position they adopt (i.e. robot ahead, robot side-by side).

The size categories in guidance sequences are single individuals, couples, triples and larger groups (Table 2). Group composition is referred to the age of the group members defining four types of group: all-children, all-young, all-adults and mixed groups.

An ad hoc coding scheme was built-up to investigate the space distribution patterns -relative position and distance between agents including the robot. 4 spatial patterns were described to classify the group spatial layout while walking with the robot: side-by-side, v-shape and leader-follower (Table 2).

\subsection{Visitors-robot face to face interaction}

According to the task description, face-to-face interaction may happen any time the robot is activated and not engaged in guiding a group. In this situation when 
typically the robot is stationary the touch screen-based interaction is available and REEM's social behavior is based on head motion and speech.

The 47 face-to-face sequences are described according to participant individual variables (genre, age), presence of other co-located visitors (group size and composition), the distance from the robot, the robot behavior (displacement, head motion) and visitor social behavior: gaze (eye contact, look at the robot, look at the screen), physical contact on robot (on the screen, other) facial expression (smile, grimaces) and gestures (wave, head motion) (See Table 3).

\section{Results}

\subsection{REEM's performance}

During the 6 day trial the robot completed 48 hours of autonomous operation walking through the museum defined space during regular public attendance at a maximum displacement speed of $4 \mathrm{~km} / \mathrm{h}$. To the best of our knowledge during this time REEM robot operated without remarkable pauses (i.e. more than one hour) and suffered a total of 5 shutdown incidences caused by visitors' misuse pressing deliberately the salient red emergency stop button placed at the robot's back.

\subsection{Group description}

The whole 96 minutes of the 91 follow-me episodes were analyzed (11.64\% of the total video-recordings where people and robot were detected together in the scene) to investigate the group walking behavior.

Concerning the group composition, $1.10 \%$ of groups that interact with the robot were all-children, $8.79 \%$ were all-youth, $52.75 \%$ were all-adults and $37.36 \%$ were mixed groups. From the mixed groups, $50 \%$ were formed by children and adults, $29.41 \%$ were formed by youth and adults, and $20.59 \%$ were formed by children, youth and adults.

Concerning the group size, $3.30 \%$ of the people walked alone with the robot, while the $96.70 \%$ arrived in groups: $10.99 \%$ of groups were couples, $14.29 \%$ triples and $71.43 \%$ larger groups.

Visitors who walked alone with the robot were $33.33 \%$ youth and $66.67 \%$ adults; couples were $10 \%$ youth, $80 \%$ adults and $10 \%$ children and adults; triples were formed by $15.38 \%$ youth, $61.54 \%$ adults, $7.69 \%$ children and adults, and $15.38 \%$ youth and adults. Larger groups were composed of $1.54 \%$ children, $6.15 \%$ youth, $46.15 \%$ adults, $23.08 \%$ children and adults, $12.31 \%$ youth and adults, and $10.77 \%$ children, youth and adults.

\subsection{Group spatial arrangement}

Results about group spatial arrangement with people walking together with robot in the follow-me behavior showed that: 
- $100 \%$ of guide-visitor couples (i.e. one person-one robot) was characterized by a leader (robot) - follower spatial arrangement (as shown in Figure 6a);

- $90 \%$ of guide-visitors triples was characterized by the robot heading the group and followed by a dyad in an inverted V-like pattern (Figure 6b), and $10 \%$ by V-like pattern (Figure $6 \mathrm{c}$ );

- $100 \%$ of four-agents groups (i.e. three people-one robot) was characterized by the robot followed by a triad (Figure 6d).

We can show that $96.15 \%$ of the formations that were analyzed have a robot leader - person follower structure, indicating a weak social cohesion between the robot and people in almost all spatial arrangements.

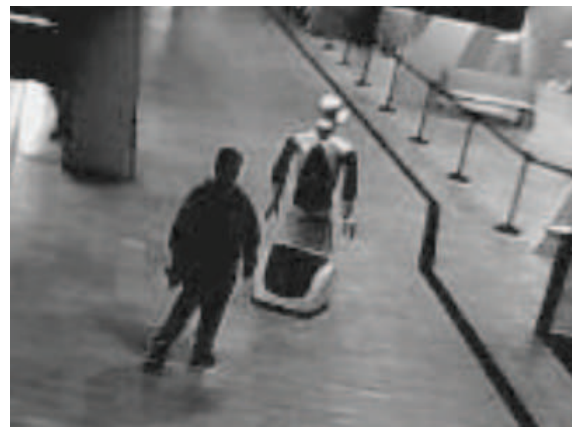

(a)

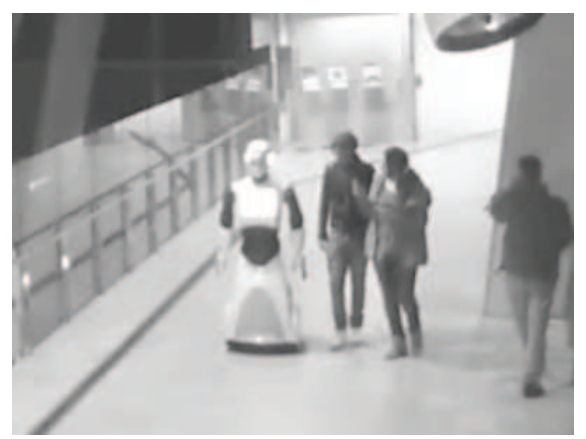

(c)

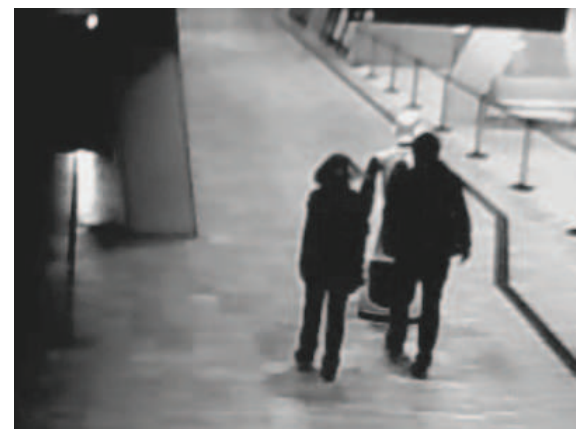

(b)

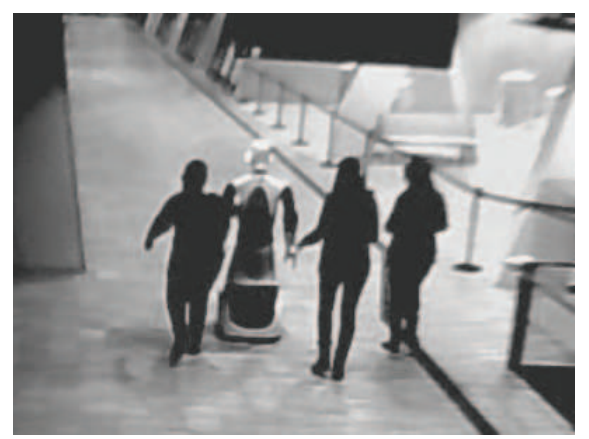

(d)

Fig. 6: Group spatial arrangements: (a) guide-visitor couple with leader-follower formation; (b) guide-visitors triple in a leader-follower spatial arrangement with robot heading the group followed by a dyad; (c) guide-visitors triple with V-like pattern; and, (d) four-agents with robot leader followed by a triad. 


\subsection{Interactive behavior}

As mentioned before, a subset of 4710 -seconds sequences of face-to-face interaction were randomly selected from the on-board recordings. The description corresponding to 42 different visitors is summarized in Table 3 in the Appendix.

In the 47 sequences the $60 \%$ of the 43 participants were males and the $30 \%$ females. $45 \%$ were adults, $17 \%$ young people and $30 \%$ children.

In 29 of the 47 sequences $(62 \%)$ the visitor established eye contact with the robot at least once (Figure $7 \mathrm{a}$ ) with a total of 50 smiles registered. In 9 occurrences the visitor kept staring at the robot's face during the whole sequence.

In 23 sequences $(62 \%)$ the visitor smile at least once with a total of 31 smiles registered. In 9 occurrences the visitor kept smiling during the whole sequence (Figure 7b).

With respect to the interface mediated communication, in 10 sequences $(21 \%)$ the visitor touched the screen at least once and in $18(38 \%)$ the user looked at the screen. Male visitors seem more prone to interact through the graphic screen: the $50 \%$ of the male visitors looked at the screen and the $29 \%$ touched the screen, in front of a $22 \%$ of female visitors that looked at the interface and only the $11 \%$ of females touched it.

In the 8 sequences showing people interacting alone with the robot (Figures $7 \mathrm{~b}-$ (d)) in 7 the visitor established eye contact and in 5 sequences they smile. There are relevant instances because the social behavior cannot be attributed to be addressed or provoked by human-human interaction (Figures $7 \mathrm{e}-(\mathrm{f})$ ).

It is noteworthy that the analyzed behaviors are not mutually exclusive. Even though in the descriptive analyses are quantified as independent behaviors, facial expressions (e.g. smile) and gestures (e.g. wave) may be presented simultaneously and actually usually are in non-verbal interpersonal communication (e.g. 100\% of observed wave behaviors appeared with smile).

\section{Discussion}

\subsection{Robot Guide's performance}

The robot succeeded in developing the role of a museum guide -attracting people, providing information and guidance- fulfilling up to 91 follow-me missions operating autonomously. Registered face to face episodes show visitors spontaneous social behavior addressed to the robot including eye contact, smiles, and greetings. Although visitors' face-to-face behavior is described qualitatively and no conclusive results can be drawn from the data some of the observed behaviors seem to account clearly for enjoyment and engagement.

However same shortcomings and difficulties were met mostly related to the challenging social context that is extremely complex, dynamic (i.e. changeable in visitors density and distribution) and sometimes crowds of visitors with uncertain trajectories. Attendance is formed by a wide range of visitors' profiles and group 
April 29, $2015 \quad 8: 28$ WSPC/INSTRUCTION $\quad$ FILE

'2013`IJHR'DiazetalV3.CameraReady 02"

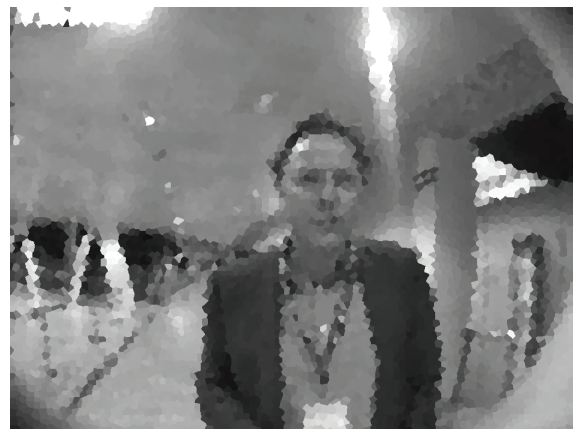

(a)

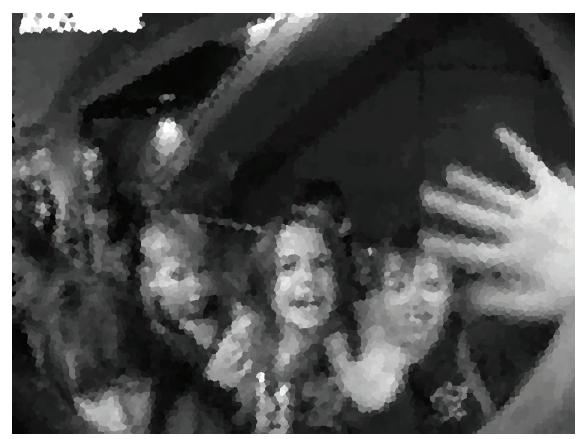

(c)

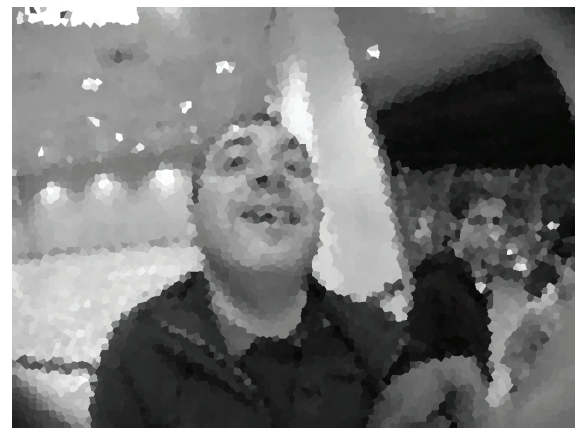

(e)

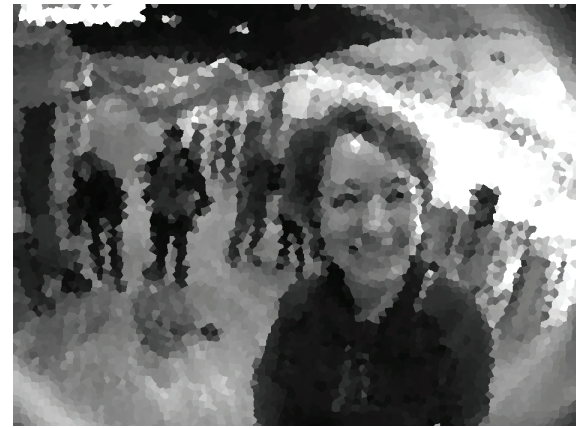

(b)

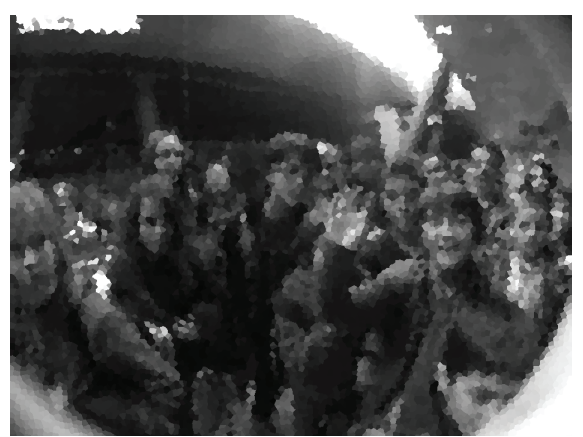

(d)

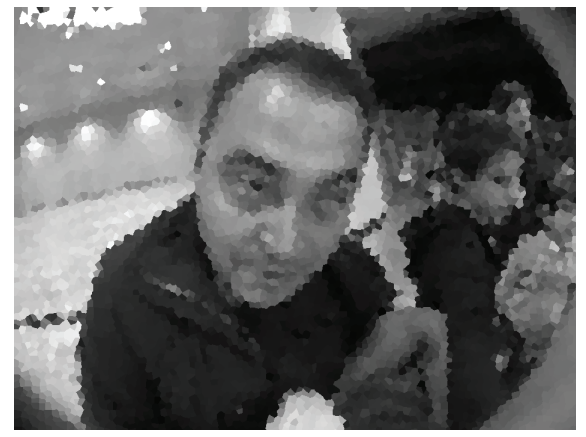

(f)

Fig. 7: Interactive behaviors: (a) visual contact; (b) smiling; (c)-(d) waving; (e)-(f) mimic head movement.

configurations with a high rate of children that increases the uncertainty. The physical scenario is not stable either due to temporary exhibitions, eventual events and maintenance tasks ${ }^{7}$.

In addition, a science museum as an experimental bed test for HRI studies has 
some peculiarities that may be outlined. The robot is an attraction itself as a piece of smart technology and an object of visitors' interest and curiosity in a context where visitors are encouraged to explore and try ${ }^{9}$. Far to become transparent in this situation the technology becomes the target and visitors do not miss the opportunity to explore and interact with the robot manipulating it (e.g. pushing the emergency button), defying its capabilities (e.g. climbing to the rear platform) and putting it in challenging situations to see what happens (e.g. activating on purpose the face tracker moving the head up and down). In our trial we have observed that eventually these visitors' active behaviors result in shutdown or the impossibility to fulfill the task.

The robot guide attracted untrained naïve people and engaged visitors 91 times in follow-me behavior without any other cue but the robot appearance and behavior, especially when it moved around, moved the head searching and tracking faces and initiated motion. The success in attracting people led the guide robot to face the "freezing robot" problem: once the environment surpasses a certain level of complexity all alternatives are unsafe and stuck in place ${ }^{15}$.

Therefore, the context of service is a challenging combination of a complex space and the willingness of people -sometimes crowds- to approach and interact with the robot. In this situation a conservative navigation for safety issues and a focus on robot's robustness is required even if it implies a sacrifice on robot's interactivity and attractiveness (e. g. discarding the communicative use of its arms). From this experience, we agree with Willeke ${ }^{8}$ that resilience for recovering from visitors' misuse -even abuse- and awkward situations (i.e. approaching a wall too closely or being crowded by people) is a crucial issue to ensure the continuity of the service.

To overcome these constrains, we consider that it would be interesting to empower the robot with some kind of authority that would be consistent with the role of guide to regulate visitors' behavior (i.e. showing people clearly what is not allowed) and to give the robot more social presence that maybe could prevent from same rough manipulations. In addition, closer but unobtrusive supervision might be provided by conductors or staff to discourage deliberate or not deliberate misuse.

\subsection{Contributions to HRI studies}

We consider that the study has a remarkable ecological validity provided that neither changes of the regular schedule of the museum nor modifications of the natural scenario was implemented -except for the set of the 2 cameras- to deploy the robot during the 6-day trial.

The literature on social space management, crowd behavior and spatiality in walking groups are revised and applied to the evaluation of hybrid groups formed by the robot and a number of naïve participants. This approach focus on groups rather than on individuals and extend the scope of HRI proxemics -mostly oriented to one-to-one interaction ${ }^{10}$ - with the consideration of dynamic spatial arrangements 
during displacement that are critical for robot's performance in public spaces.

The detailed description of the HRI episodes offers empirical based insights that can be of interest to improve the evaluation and design of HRI in public and dense environments and to focus on relevant variables like the social situation, the role the robot's takes, its social affordances, the actual robot's behavior and the physical constrains.

Furthermore, this study has outlined the feasibility and convenience of automatic processing techniques (e.g. computer vision) to study spatial HRI through systematic observation even though their use still represents great challenges of reliability and robustness in the wild.

\subsection{Limitations and Future work}

\subsubsection{Camera coverage}

The two cameras do not cover in detail all the relevant interactive behaviors between robot and visitors in the experimental space. In particular, even though the perspective provided was enough for group arrangements classification, face-to-face interaction at the end of the guidance episodes are missed being the destination points too far away from the cameras. Adding another on-board omnidirectional camera could provide a view of all the social space around the robot and facilitate the use of automatic spatial behavior analyses.

\subsubsection{Face-to-face interaction data}

Due to technical constraints it was not possible to videotape continuously face-toface interaction from the on-board subjective-perspective camera so the analyses has been done on a sample of sequences from the 83 minutes of available recordings. No systematic or representative conclusions can be drawn from data. Nevertheless the observational data analyses provide a sound base for an empirical-based contextual coding scheme for further research.

\subsubsection{Measures of density}

One very relevant variable that influences group walking behavior is the space density, which has not been measured accurately in our study. Further studies must provide density measures preferably automatically obtained from vision processing.

\subsubsection{Logging of Robot behavior and interaction on GUI}

To triangulate human-robot interaction evaluation it would be very interesting to analyse the logs of robot behavior and of the interface-based interaction. Data are in principle available from robot's log but it must be faced the issues of processing and storage. 


\subsubsection{Visitors' experience}

The evaluation of the guide robot performance would benefit from a complementary assessment of visitors experience from short questionnaires after interacting with the robot. This self-report data could be of great interest to interpret or contrast the observational data.

\subsubsection{Factors influencing behavior}

While the aim of this study is to provide descriptive analyses and evidence-based insight with high ecological validity, systematic exploration of factors influencing the observed behavior should be carried out in future works to better understand the interactive behavior in this context. Systematic quantitative studies on individual variables (i.e. gender, age), social context, robot personality (i.e. body language), and group features (i.e. composition, density, dispersion and velocity) could be of the greatest interest in social HRI research.

\section{Conclusions}

An exploratory study on group-robot interaction was carried out during a week in an open and natural environment to observe visitors' spatial behavior and communication with the guide robot REEM in a popular science museum.

The robot succeeded in developing the role of a museum guide -attracting people, providing information and guidance- fulfilling up to 91 follow-me missions operating autonomously. Registered face to face episodes show untrained visitors social behavior addressed to the robot including eye contact, smiles, and greetings.

Differently from previous works on mobile service robots that evaluate navigation and HRI as separate functions we address spatial behavior analyses focusing on its social meaning, not only as a prerequisite for effective communication (i.e. orientation, positioning) but as potential communicative acts (i.e. express intent and emotions).

The analysis is focused on visitors' groups rather than individual. Groups were described according to their composition, size, spatial formations and interactive behavior with the robot during guidance. Observational methods applied to evaluate group-robot interaction provide fruitful insight to understand the relationship between robot positioning and effcient communication (i.e. walking side-by-side) and

between robot motion cues (e.g. gaze behavior, body orientation) and collaborative walking together behavior through populated environments.

\section{Acknowledgements}

This research was supported in part by the PATRICIA Research Project (TIN201238416-C03-01,03), funded by the Spanish Ministry of Economy and Competitiveness. The authors would like to express their very great appreciation to CosmoCaixa 
Science Museum Barcelona and Fundació "La Caixa" for providing the research facilities used in this study. They are particularly grateful for the encouraging support of Ms. Cristina Smandia from the Communication and Audiences Department, Fundació "La Caixa". Assistance provided by Mr. Jordi-Ysard Puigbò, Ms. Judit Casacuberta (Technical University of Catalonia), Ms. Isaura Almar, and Mr. Miguel Kaouk (La Salle Engineering) is greatly appreciated. Finally, special thanks are extended to Dr. Ricardo Téllez and the technical staff of PAL Robotics company.

\section{References}

1. M. Kuderer, H. Kretzschmar and W. Burgard, Teaching mobile robots to cooperatively navigate in populated environments, in IEEE/RSJ Int. Conf. Intelligent Robots and Systems (IROS) (IEEE Press, Tokyo, Japan, 2013), pp. 3138-3143.

2. C.L. Bethel and R.R. Robin, Affective expression in appearance constrained robots, in ACM/IEEE Int. Conf. Human-Robot Interaction (HRI) (ACM Press, Salt Lake City, Utah, 2006), pp. 327-328.

3. H. Huettenrauch, K. Severinson Eklundh, A. Green and E.A. Topp, Investigating spatial relationships in human-robot interaction, in Int. Conf. Intelligent Robots and Systems (IROS) (IEEE Press, Beijing, China, 2006), pp. 5052-5059.

4. T. Kanda, T. Hirano, D. Eaton and H. Ishiguro, Interactive robots as social partners and peer tutors for children: a field trial, Human-Computer Interaction 19(1) (2004) $61-84$.

5. M. Montemerlo, J. Pineau, N. Roy, S. Thrun and V. Verma, Experiences with a mobile robotic guide for the elderly, in Nat. Conf. Artificial Intelligence (AAAI) (AAAI Press, Edmonton, Alberta, Canada, 2002), pp. 587-592.

6. R. Siegwart, K.O. Arras, S. Bouabdallah, D. Burnier, G. Froidevaux, X. Greppin, B. Jensen, A. Lorotte, L. Mayor, M. Meisser, R. Philippsen, R. Piguet, G. Ramel, G. Terrien and N. Tomatis, Robox at Expo.02: a large-scale installation of personal robots, Robotics and Autonomous Systems 42(3) (2003) 203-222.

7. W. Burgard, A.B. Cremers, D. Fox, D. Hähnel, G. Lakemeyer, D. Schulz, W. Steiner and S. Thrun, The interactive museum tour-guide robot, in Nat. Conf. Artificial Intelligence (AAAI) (AAAI Press, Madison, Wisconsin, 1998), pp. 11-18.

8. T. Willeke, C. Kunz and I.R. Nourbakhsh, The history of the mobot museum robot series: an evolutionary study, in Florida Artif. Intell. Res. Soc. Conf. (FLAIRS) (Key West, Florida, 2001), pp. 514-518.

9. M. Shiomi, T. Kanda, H. Ishiguro and N. Hagita, Interactive humanoid robots for a science museum, IEEE Intelligent Systems 22(2) (2007) 25-32.

10. A. Kristoffersson, K. Severinson Eklundh and A. Loutfi, Measuring the quality of interaction in mobile robotic telepresence: a pilots perspective, International Journal of Social Robotics 5(1) (2012) 89-101.

11. P. Marshall, Y. Rogers and N. Pantidi, Using F-formations to analyse spatial patterns of interaction in physical environments, in ACM Conf. Comp. Supp. Coop. Work (CSCW) (ACM Press, Hangzhou, China, 2011), pp. 445-454.

12. A. Kendon, Spacing and orientation in co-present interaction, in Development of Multimodal Interfaces: Active Listening and Synchrony, Series in Lecture Notes in Computer Science, Vol. 5967 (Springer Berlin Heidelberg, 2010), pp. 1-15.

13. A. Bauer, D. Wollherr and M. Buss, Human-robot collaboration: A survey, International Journal of Humanoid Robotics 5(1) (2008).

14. P. Ratsamee, Y. Mae, K. Ohara, T. Takubo and T. Arai, Human-robot collision 
avoidance using a modified social force model with body pose and face orientation, International Journal of Humanoid Robotics 10(1) (2013) 1-24.

15. P. Trautman and A. Krause, Unfreezing the robot: navigation in dense, interacting crowds, in IEEE/RSJ Int. Conf. Intelligent Robots and Systems (IROS) (IEEE Press, Taipei, Taiwan, 2010), pp. 797-803.

16. D. Karreman, L. Utama, M. Joosse, M. Lohse, B. van Dijk and V. Evers, Robot etiquette: how to approach a pair of people?, in ACM/IEEE Int. Conf. Human-Robot Interaction (HRI) (ACM Press, Bielefeld, Germany, 2014), pp. 196-197.

17. S. Thrun, M. Bennewitz, W. Burgard, A.B. Cremers, F. Dellaert, D. Fox, D. Hahnel, C. Rosenberg, N. Roy, J. Schulte and D. Schulz, MINERVA: a second-generation museum tour-guide robot, in IEEE Int. Conf. Robotics and Automation (ICRA) (IEEE Press, Detroit, Michigan, 1999), pp. 1999-2005.

18. D.J. Feil-Seifer and M.J. Matarić, Distance-based computational models for facilitating robot interaction with children, Journal of Human-Robot Interaction 1(1) (2012) $55-77$.

19. E. Torta, R. Cuijpers, H. Raymond, J.F. Juola and D. Van Der Pol, Modeling and testing proxemic behavior for humanoid robots, International Journal of Humanoid Robotics 9(4) (2012).

20. H. Kuzuoka, Y. Suzuki, J. Yamashita and K. Yamazaki, Reconfiguring spatial formation arrangement by robot body orientation, in ACM/IEEE Int. Conf. Human-Robot Interaction (HRI) (ACM Press, Osaka, Japan, 2010), pp. 285-292.

21. A. Garrell and A. Sanfeliu, Cooperative social robots to accompany groups of people, International Journal of Robotics Research 31(13) (2012) 1675-1701.

22. S. Bandini, A. Gorrini and G. Vizzari, Towards an integrated approach to crowd analysis and crowd synthesis: a case study and first results, Pattern Recognition Letters 44(July) (2014) 16-29.

23. M. Costa, Interpersonal distances in group walking, Journal of Nonverbal Behavior 34(1) (2010) 15-26.

24. A. Beck, L. Cañamero, A. Hiolle, L. Damiano, P. Cosi, F. Tesser and G. Sommavilla, Interpretation of emotional body language displayed by a humanoid robot: a case study with children, International Journal of Social Robotics 5(3) (2013) 325-334.

25. CosmoCaixa, General information about CosmoCaixa Barcelona, http://obrasocial.lacaixa.es/laCaixaFoundation/home_en.html [accessed on 16 March 2015].

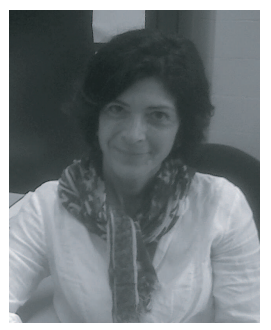

Marta Díaz-Boladeras received her B.Sc/M.Sc degree in Psychology from the University of Barcelona (UB) (1984), the Ph.D. in Business Administration from the Department of Management at the Universitat Politècnica de Catalunya - BarcelonaTech (UPC) (1997) and the Ms.Sc degree in Occupational Risk Prevention / Ergonomics and Applied Psycho-Sociology from the UPC (2000). She is currently engaged in the Doctoral Program on Primatology and Ethology at the Faculty of Psychology (UB). In 1990 joined the Department of Management at the UPC and became full professor in 1997. Director of the IBuX-Lab at the Technical Research Centre for Dependency Care and Autonomous Living (UPC). Her research interests are social human-robot interaction and ICT systems design for health and well-being. Her current research focuses on the application of social psychology and 
ethology models and methods to long-term human-robot interaction optimisation.

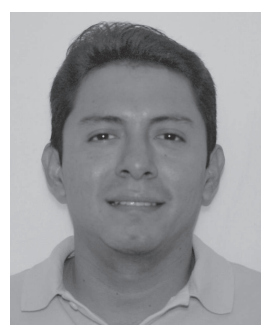

Dennys Paillacho received his B.S. degree in Computer Science from the Escuela Superior Politécnica del Litoral in 2003 and his M.S. degree in Automatic Control and Robotics from Universitat Politècnica de Catalunya BarcelonaTech in 2010. From 2001 to 2004 Mr. Paillacho was a software engineer with Sonda Technology Group in Ecuador. From 2004 he was as Associate Professor and Research Assistant at Escuela Superior Politécnica del Litoral. Currently, Mr. Paillacho is a Ph.D. candidate with the Department of Automatic Control (ESAII) at Universitat Politècnica de Catalunya in Barcelona. His interests include social robotics, human-robot spatial interaction and educational robotics.

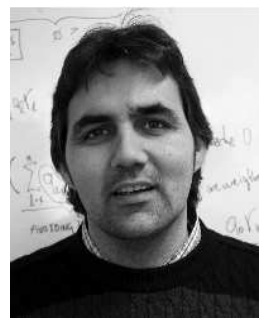

Cecilio Angulo received his M.S. degree in Mathematics from the University of Barcelona, Spain, and his Ph.D. degree in Sciences from the Universitat Politècnica de Catalunya BarcelonaTech, Spain, in 1993 and 2001, respectively. From 1999 to 2007, he was at the Universitat Politècnica de Catalunya, as Assistant Professor. He is nowadays an Associate Professor in the Department of Automatic Control, in the same university. From 2011 he also served as Director of the Master's degree in Automatic Control and Robotics. He's currently the Director of the Knowledge Engineering Research Group where he is responsible for research projects in the area of social cognitive robotics.

Cecilio Angulo is the author of over 250 technical publications. His research interests include cognitive robotics, machine learning algorithms and social robotics applications.

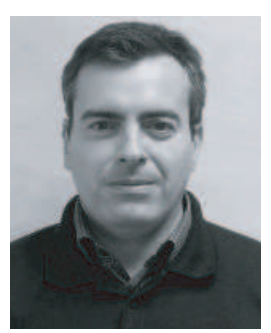

Oriol Torres received his M.S. degree in Electronic Engineering from the Universitat Politècnica de Catalunya BarcelonaTech, Spain, in 1999. From 2000 to 2001, he worked in Xilinx, Inc., the worldwide FPGA-leader company. From 2001 to 2004, he was at the Universitat Politècnica de Catalunya as Research Assistant. In 2004, he started the company PAL Robotics, where he occupied different positions such as Electronics Manager and Project Manager. In 2010, he also completed a Master in Project Management. He is currently Business Strategy Manager from PAL Robotics. His research interests include robotic 
April 29, $2015 \quad 8: 28$ WSPC/INSTRUCTION $\quad$ FILE

'2013.IJHR' DiazetalV3. CameraReady 02"

Evaluating Group-Robot Interaction in Crowded Public Spaces

topics and human machine interaction.

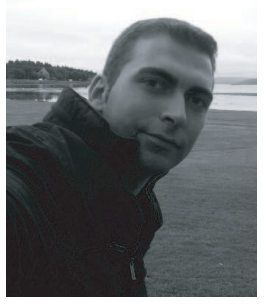

Jonathan González-Diéguez received his M.Sc., degree in Telecommunications Engineering, specialized on Communication Systems, from the Universitat Politècnica de Catalunya - BarcelonaTech, Spain, in 2012. Since then, he joined PAL Robotics managing different areas of the company. He is responsible for REEM robot in real environment conditions.

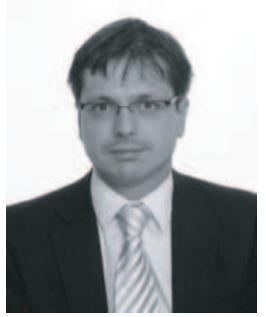

Jordi Albo-Canals received his B.Sc, M.Sc., and PhD degree in Telecommunications Engineering from the Engineering School of La Salle - Ramon Llull University of Barcelona, Spain, in 2000, 2003, and 2012, respectively. Currently, he is an Associate Professor in the Electrical Engineering Department of La Salle - Ramon Llull University (Spain), the Robotics Director of La Salle Almere (Netherlands), and a Visiting Professor in the Mechanical Engineering Department at Tufts University (US). His research interests include theoretical and applied aspects of human factors in robot design and cloud robotics. 


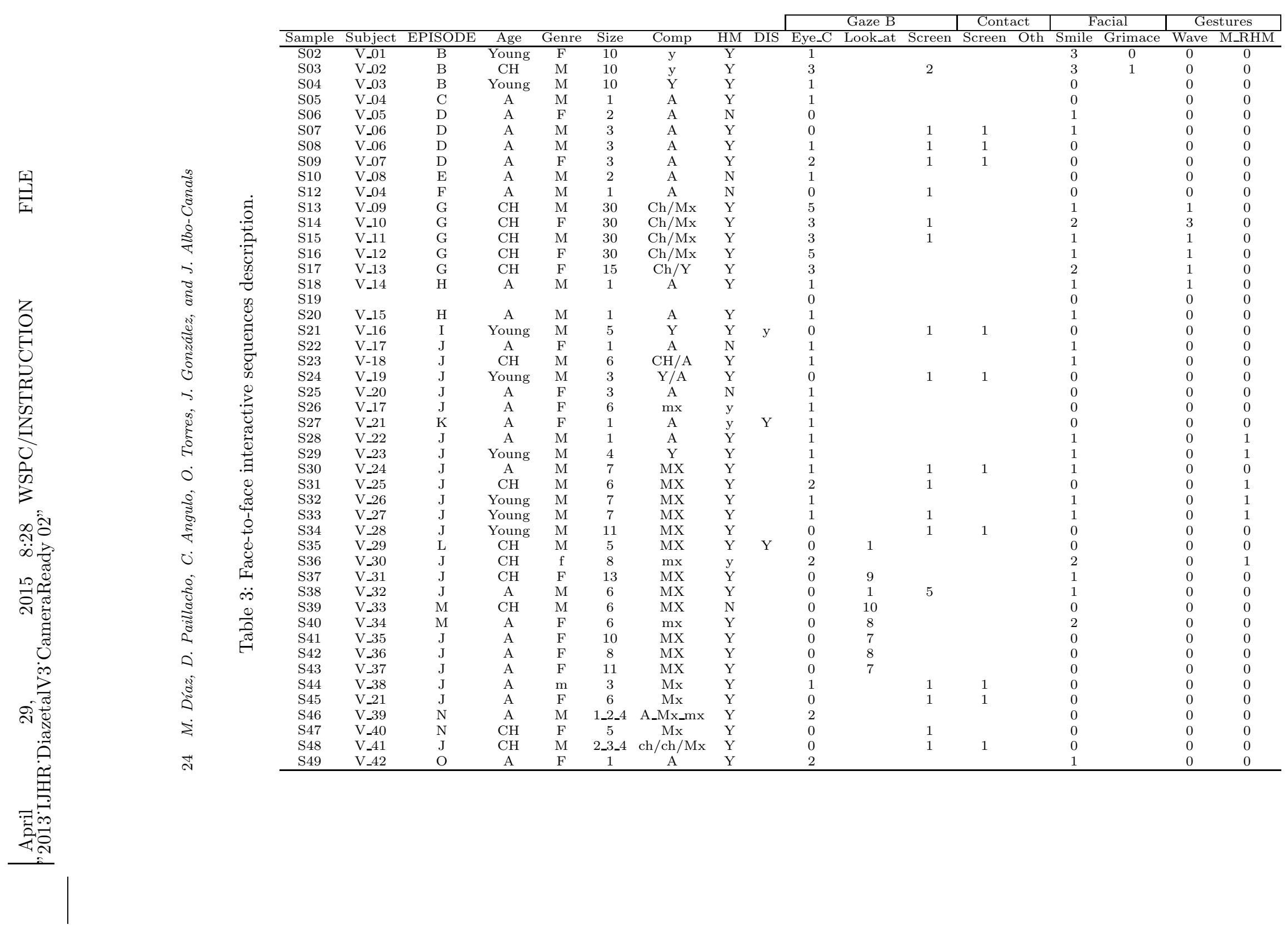

